\title{
BOUNDARY BEHAVIOR OF INVARIANT FUNCTIONS ON PLANAR DOMAINS
}

\author{
NIKOLAI NIKOLOV, MARIA TRYBUŁA, AND LYUBOMIR ANDREEV \\ AbStract. Precise behavior of the Carathéodory, Kobayashi and \\ Bergman metrics and distances near smooth boundary points of \\ planar domains is found under different assumptions of regularity.
}

The most precise known estimates on the boundary behavior of the Carathéodory, Kobayashi and Bergman metrics $\left(\gamma_{D}, \kappa_{D}\right.$ and $\left.\beta_{D}\right)$ of a bounded strictly pseudoconvex domain $D$ in $\mathbb{C}^{n}$ with $\mathcal{C}^{3}$ - or $\mathcal{C}^{4}$-smooth boundary can be found in [2, 3, 6] (for the $C^{2, \varepsilon}$-smooth case see [8]).

Set $d_{D}(z)=\operatorname{dist}(z, \partial D)$.

For $D \subset \mathbb{C}$ let $\gamma_{D}(z)=\gamma_{D}(z ; 1)$, etc., and

$$
m_{D} \in A=\left\{\gamma_{D}, \kappa_{D}, \beta_{D} / \sqrt{2}, \sqrt{\pi K_{D}}\right\},
$$

where $K_{D}$ is the Bergman kernel on the diagonal $\left(\beta_{D}\right.$ is defined if $\mathbb{C} \backslash D$ is not polar). Note that the functions in $A$ coincide if $D \subsetneq \mathbb{C}$ is simply connected.

In the plane the mentioned above results can be read as follows:

1. If $D \Subset \mathbb{C}$ has $C^{2, \varepsilon}$-smooth boundary $(\varepsilon \in(0,1])$, then the function $\frac{2 m_{D} d_{D}-1}{d_{D}^{\varepsilon / 2}}$ is bounded;

2. If $D \Subset \mathbb{C}$ has $C^{4}$-smooth boundary, then $m_{D}-\frac{1}{2 d_{D}}$ is bounded.

Our first goal is to obtain the precise behavior of $m_{D}$ near $\mathcal{C}^{2}$-smooth boundary points of domains in $\mathbb{C}$.

Proposition 1. If a is a $\mathcal{C}^{2}$-smooth boundary point of a domain $D \subset \mathbb{C}$, then

$$
\lim _{z \rightarrow a}\left(m_{D}(z)-\frac{1}{2 d_{D}(z)}\right)=\frac{\chi_{D}(a)}{4},
$$

where $\chi_{\partial D}(a)$ is the signed curvature of $\partial D$ at a.

2010 Mathematics Subject Classification. 32F45, 32A25.

Key words and phrases. Carathéodory metric and distance, Kobayashi metric and distance, Bergman kernel, metric and distance.

M. Trybula and L. Andreev are partially supported by the Bulgarian National Science Found under contract DFNI-I 02/14. 
We have a weaker result in the $\mathcal{C}^{1}$-smooth case.

Proposition 2. (see [4]) If a is a $\mathcal{C}^{1}$-smooth boundary point of a domain $D \subset \mathbb{C}$, then

$$
\lim _{z \rightarrow a} 2 m_{D}(z) d_{D}(z)=1 .
$$

The following intermediate result holds in the $\mathcal{C}^{1, \varepsilon}$-smooth case.

Proposition 3. If $\varepsilon \in(0,1]$ and $a$ is a $\mathcal{C}^{1, \varepsilon}-$ smooth boundary point of a domain $D \subset \mathbb{C}$, then $\frac{2 m_{D} d_{D}-1}{d_{D}^{\varepsilon}}$ is a bounded function near a.

This result is sharp as the next example shows.

Example 4. (a) If $\varepsilon \in(0,1)$, then $f(z)=z-\frac{z^{1+\varepsilon}}{4}$ is a univalent function on $\mathbb{D}_{1}=\{z \in \mathbb{C}:|z-1|<1\}, D=f\left(\mathbb{D}_{1}\right)$ has $\mathcal{C}^{1, \varepsilon}$-smooth boundary and

$$
\lim _{u \rightarrow 0+} \frac{2 m_{D}(u) d_{D}(u)-1}{d_{D}^{\varepsilon}(u)}=\frac{\varepsilon}{4} .
$$

(b) The domain $D=\mathbb{D} \cup\{z \in \mathbb{C}:|x|<1, y>0\}$ is $\mathcal{C}^{1,1}$-smooth and

$$
\liminf _{z \rightarrow 1}\left(2 m_{D}(z)-\frac{1}{d_{D}(z)}\right)=0<\frac{1}{4}=\limsup _{z \rightarrow 1}\left(2 m_{D}(z)-\frac{1}{d_{D}(z)}\right) .
$$

Combining the proofs of Propositions 1 and 3 , one may obtain the following result in the $\mathcal{C}^{2, \varepsilon}$-smooth case whose proof we omit.

Proposition 5. If $\varepsilon \in(0,1)$ and $a$ is a $\mathcal{C}^{2, \varepsilon}$-smooth boundary point of a domain $D \subset \mathbb{C}$, then $\frac{4 m_{D}-2 / d_{D}-\chi_{D}}{d_{D}^{\varepsilon}}$ is a bounded function near $a$, where $\chi_{D}(z)$ is the signed curvature of $\partial D$ at the closest point to $z$.

A similar example to Example 4 shows that this result is sharp.

Recall now that the boundary behavior of Kobayashi distance $k_{D}$ of a bounded strictly pseudoconvex domain $D$ in $\mathbb{C}^{n}$ is known up to \pm constant (see [1]). The same behavior have the Carathéodory distance $c_{D}$, the Lempert function $l_{D}$ and, in the $\mathcal{C}^{2, \varepsilon}$-smooth case, the Bergman distance $b_{D}$ divided by $\sqrt{n+1}$ (see [8]).

Sharper estimates of these invariants in the case of Dini-smooth bounded domains in $\mathbb{C}$ can be found in [7, 10] $\left(k_{D}=l_{D}\right.$ if $\left.D \subset \mathbb{C}\right)$. Our next aim is to find the precise behavior of $p_{D} \in\left\{c_{D}, k_{D}, b_{D} / \sqrt{2}\right\}$ near Dini-smooth boundary points of domains in $\mathbb{C}$ (these distances coincide if $D \subsetneq C$ is simply connected). Recall that Dini-smoothness is between $\mathcal{C}^{1}$ - and $\mathcal{C}^{1, \varepsilon}$-smoothness. 
Set $s_{D}(z, w):=\sinh ^{-1} \frac{|z-w|}{2 \sqrt{d_{D}(z) d_{D}(w)}}$

$$
=\log \frac{|z-w|+\sqrt{|z-w|^{2}+4 d_{D}(z) d(w)}}{2 \sqrt{d_{D}(z) d_{D}(w)}} .
$$

Note that $s_{D}=p_{D}$ if $D \subset \mathbb{C}$ is a half-plane.

Proposition 6. If a is a Dini-smooth boundary point of a domain $D \subset \mathbb{C}$, then

$$
\lim _{z, w \rightarrow a}\left(p_{D}(z, w)-s_{D}(z, w)\right)=0 .
$$

When $s_{D} \rightarrow 0$, Proposition 6 can be improved even in the $\mathcal{C}^{1}$-smooth case.

Proposition 7. (a) If a is a $\mathcal{C}^{1}$-smooth boundary point of a domain $D \subset \mathbb{C}$, then

$$
\lim _{\substack{z, w \rightarrow a \\ z \neq w}} \frac{p_{D}(z, w)}{s_{D}(z, w)}=1 .
$$

(b) If $D \subset \mathbb{C}$ is a $\mathcal{C}^{1}$-smooth bounded domain, then

$$
\lim _{z \rightarrow \partial D} \frac{p_{D}(z, w)}{s_{D}(z, w)}=1 \quad \text { uniformly in } w \neq z .
$$

\section{Definitions}

1. A boundary point $p$ of a domain $D \subset C$ is said to be Dini-smooth if $\partial D$ near $p$ is given by a Dini-smooth curve $\gamma:[0,1] \rightarrow \mathbb{C}$ with $\gamma^{\prime} \neq 0$ (i.e. $\int_{0}^{1} \frac{\omega(t)}{t} d t<\infty$, where $\omega$ is the modulus of continuity of $\gamma^{\prime}$ ). A domain in $\mathbb{C}$ is called Dini-smooth if all its boundary points are Dini-smooth.

2. Let $D$ be a domain in $\mathbb{C}^{n}$. The Kobayashi (pseudo)distance $k_{D}$ is the largest (pseudo)distance not exceeding the Lempert function

$$
l_{D}(z, w)=\inf \left\{\tanh ^{-1}|\alpha|: \exists \varphi \in \mathcal{O}(\mathbb{D}, D) \text { with } \varphi(0)=z, \varphi(\alpha)=w\right\},
$$

where $\mathbb{D}$ is the unit disc. Note that $k_{D}$ is the integrated form of the Kobayashi (pseudo)metric

$$
\kappa_{D}(z ; X)=\inf \left\{|\alpha|: \exists \varphi \in \mathcal{O}(\mathbb{D}, D) \text { with } \varphi(0)=z, \alpha \varphi^{\prime}(0)=X\right\} .
$$

Denote by $c_{D}$ the Carathéodory (pseudo)distance of $D$ :

$$
c_{D}(z, w)=\sup \left\{\tanh ^{-1}|\psi(w)|: \psi \in \mathcal{O}(D, \mathbb{D}) \text { with } \psi(z)=0\right\} .
$$

Then $c_{D} \leq k_{D} \leq l_{D}$. 
The Bergman distance $b_{D}$ of $D$ is the integrated form of the Bergman metric $\beta_{D}$, i.e.

$$
b_{D}(z, w)=\inf _{\gamma} \int_{0}^{1} \beta_{D}\left(\gamma(t) ; \gamma^{\prime}(t)\right) d t, \quad z, w \in D,
$$

where the infimum is taken over all smooth curves $\gamma:[0,1] \rightarrow D$ with $\gamma(0)=z$ and $\gamma(1)=w$.

Recall that

$$
\beta_{D}(z ; X)=\frac{M_{D}(z ; X)}{\sqrt{K_{D}(z)}}, \quad z \in D, X \in \mathbb{C}^{n},
$$

where

$$
M_{D}(z ; X)=\sup \left\{\left|f^{\prime}(z) X\right|: f \in L_{h}^{2}(D),\|f\|_{L^{2}(D)} \leq 1, f(z)=0\right\}
$$

and

$$
K_{D}(z)=\sup \left\{|f(z)|^{2}: f \in L_{h}^{2}(D),\|f\|_{L^{2}(D)} \leq 1\right\}
$$

is the Bergman kernel on the diagonal (we assume that $K_{D}(z)>0$ ).

Note that $M_{D} \leq M_{G}$ and $K_{D} \leq K_{G}$ if $G$ is a subdomain of $D$ but $\beta_{D}$ does not share this property, in general.

It is well-known that $c_{D} \leq b_{D}$.

We refer to [5] for other properties of the above invariants.

\section{Proofs}

Proof of Proposition 1 . For $z \in D$ near a there is a unique $\mathcal{C}^{2}$-smooth point $z^{\prime} \in \partial D$ such that $\left|z^{\prime}-z\right|=d_{D}(z)$. Denote by $n_{z^{\prime}}$ the inner unit normal vector to $\partial D$ at $z^{\prime}$ and set $\phi_{z}(\tau)=z^{\prime}+\tau n_{z^{\prime}}(\tau \in \mathbb{C})$, $D_{z}=\phi_{z}^{-1}(D)$.

For any $\varepsilon>0$ there exists $\delta>0$ such that if $|z-a|<\delta$, then

$$
D^{\varepsilon, \delta}:=D^{\varepsilon} \cap \delta \mathbb{D} \subset D_{z} \subset D^{-\varepsilon} \cup(\mathbb{C} \backslash \delta \overline{\mathbb{D}})=: D^{-\varepsilon, \delta},
$$

where $D^{ \pm \varepsilon}=\left\{\tau \in \mathbb{C}: 2 \operatorname{Re} \tau>\chi^{ \pm \varepsilon}|\tau|^{2}\right\}$ and $\chi^{ \pm \varepsilon}=\chi_{\partial D}(a) \pm \varepsilon$. Note that $D^{ \pm \varepsilon}$ is a disc, the complement of a disc or a half-plane.

Since $\beta_{D}=\frac{M_{D}}{\sqrt{K_{D}}}$, to prove the result, we may replace $\beta_{D} / \sqrt{2}$ in $A$ by $\sqrt{c M_{D}}$, where $c=\sqrt{\pi / 2}$. Then

$$
m_{D^{-\varepsilon, \delta}}\left(d_{D}(z)\right) \leq m_{D}(z) \leq m_{D^{\varepsilon, \delta}}\left(d_{D}(z)\right) .
$$

Lemma L. (localization) Let $\Pi$ be the upper half-plane. Then

$$
0 \leq m_{\Pi \cap \mathbb{D}}(z)-m_{\Pi}(z) \leq \frac{|z|}{1-|z|^{2}} .
$$


Subproof. The map $f: z \mapsto\left(\frac{z+1}{z-1}\right)^{2}$ transforms conformally $\Pi \cap \mathbb{D}$ onto П. Hence

$$
\begin{aligned}
m_{\Pi \cap \mathbb{D}}(z)-m_{\Pi}(z) & =\frac{\left|f^{\prime}(z)\right|}{2 \operatorname{Im} f(z)}-\frac{1}{2 \operatorname{Im} z}=\frac{1}{2 \operatorname{Im} z}\left(\frac{\left|1-z^{2}\right|}{1-|z|^{2}}-1\right) \\
& \leq \frac{\left.\left|z^{2}-\right| z\right|^{2} \mid}{2 \operatorname{Im} z\left(1-|z|^{2}\right)}=\frac{|z|}{1-|z|^{2}} .
\end{aligned}
$$

Lemma L easily implies that $m_{D^{ \pm \varepsilon, \delta}}(\tau)-m_{D^{ \pm \varepsilon}}(\tau) \rightarrow 0$ as $\tau \rightarrow 0$. Simple calculations show that $m_{D^{ \pm \varepsilon}}(\tau)-\frac{1}{2 \tau} \rightarrow \frac{\chi^{ \pm \varepsilon}}{4}$ as $\tau \rightarrow 0+$. Thus $\frac{\chi^{-\varepsilon}}{4} \leq \liminf _{z \rightarrow a}\left(m_{D}(z)-\frac{1}{2 d_{D}(z)}\right) \leq \limsup _{z \rightarrow a}\left(m_{D}(z)-\frac{1}{2 d_{D}(z)}\right) \leq \frac{\chi^{\varepsilon}}{4}$.

It remains to let $\varepsilon \rightarrow 0$.

Proof of Proposition 3. We may replace $D^{ \pm \varepsilon}$ from above by

$$
D^{ \pm \varepsilon}=\left\{z \in \mathbb{C}: x> \pm c|y|^{1+\varepsilon}\right\}
$$

where $c>0$ is a constant.

If $\varepsilon=1$, it follows as before that

$$
-\frac{c}{2} \leq \liminf _{z \rightarrow a}\left(m_{D}(z)-\frac{1}{2 d_{D}(z)}\right) \leq \limsup _{z \rightarrow a}\left(m_{D}(z)-\frac{1}{2 d_{D}(z)}\right) \leq \frac{c}{2} .
$$

Otherwise, we may choose a conformal map $\eta_{ \pm}$from $D^{ \pm \varepsilon}$ onto the right half-plane $\Pi$ that extends $\mathcal{C}^{1+\varepsilon_{-}}$-smoothly at 0 such that $\eta_{ \pm}(0)=0$ and $\eta_{ \pm}\left(\mathbb{R}^{+}\right)=\mathbb{R}^{+}$. Using Lemma $\mathrm{L}$, it is enough to show that

$$
g(x)=\frac{2 x m_{D^{ \pm \varepsilon}}(x)-1}{x^{\varepsilon}}
$$

is a bounded function on $(0,1)$ which follows from the equalities

$$
g(x)=\frac{x \eta_{ \pm}^{\prime}(x)-\eta_{ \pm}(x)}{x^{\varepsilon} \eta_{ \pm}(x)}=\frac{x}{\eta_{ \pm}(x)} \cdot \frac{\eta_{ \pm}^{\prime}(x)-\eta_{ \pm}^{\prime}\left(\xi_{x}\right)}{x^{\varepsilon}}, \quad \xi_{x} \in(0, x) .
$$

Proof of Example 4. (a) The univalence of $f$ follows from the inequality $\operatorname{Re} f^{\prime}>0$ on $\mathbb{D}_{1}$ (cf. [11, Proposition 1.10]). The $\mathcal{C}^{1, \varepsilon}$-smoothness of $\partial D$ is clear.

Choose now $c>0$ such that $D \supset G=\left\{u+i v \in c \mathbb{D}: c^{1-\varepsilon} u>|v|^{1+\varepsilon}\right\}$. Set $\delta=\frac{1+\varepsilon}{1-\varepsilon}$. It is not difficult to compute that

$$
\lim _{u \rightarrow 0+} \frac{u-d_{G}(u)}{u^{\delta}}=\frac{2}{c^{2}(1+\varepsilon)^{\delta}(1-\varepsilon)}
$$


and hence

$$
\lim _{u \rightarrow 0+} \frac{u-d_{D}(u)}{u^{1+\varepsilon}}=0
$$

Let $x=f^{-1}(u)$. Since $m_{D}(u) f^{\prime}(x)=m_{\mathbb{D}_{1}}(x)$, then

$$
\lim _{u \rightarrow 0+} \frac{2 m_{D}(u) d_{D}(u)-1}{d_{D}^{\varepsilon}(u)}=\lim _{x \rightarrow 0+} \frac{\frac{2}{x(2-x)} \cdot \frac{4 x-x^{1+\varepsilon}+o\left(x^{1+\varepsilon}\right)}{4-(1+\varepsilon) x^{\varepsilon}}-1}{x^{\varepsilon}}=\frac{\varepsilon}{4} .
$$

(b) Let $u$ and $v$ be the respective liminf and limsup. Then $u \geq 0$ by convexity and $v \leq 1 / 4$, since $D$ satisfies the interior 1 -ball condition. On the other hand, Lemma L easily implies that if $a, b \in \partial D \backslash\{ \pm 1\}$ are such that $|\operatorname{Re} a|=1$ and $|b|=1$, then $\lim _{z \rightarrow a} m_{D}(z)=0$ and $\lim _{z \rightarrow b} m_{D}(z)=$ $1 / 4$ which shows that $u \leq 0$ and $v \geq 1 / 4$.

Proof of Proposition 6 for $c_{D}$ and $k_{D}$. We shall use arguments from the proof of [7, Proposition 5].

We may find a Dini-smooth Jordan curve $\zeta$ such that $\zeta=\partial D$ near $a$ and $D \subset \zeta_{\text {ext }}$. Let $b \notin \overline{\zeta_{\text {ext }}}, \varphi(z)=\frac{1}{z-b}$ and $G=\zeta_{\text {ext }} \cup\{0\}$. Let $\psi: G \rightarrow \mathbb{D}$ be a Riemann map. It extends to a $C^{1}$-diffeomorphism from $\bar{G}$ to $\overline{\mathbb{D}}$ (cf. [11, Theorem 3.5]). Let $\theta$ maps conformally $\mathbb{D}$ onto a half-plane $\Pi$. Setting $\eta=\theta \circ \psi \circ \varphi$, then

$$
c_{D}(z, w) \geq c_{\Pi}(\eta(z), \eta(w))=s_{\Pi}(\eta(z), \eta(w)) .
$$

Since $\lim _{z \rightarrow a} \frac{d_{\Pi}(\eta(z))}{d_{D}(z)}=\left|\eta^{\prime}(a)\right|$, it follows that

$$
\lim _{z, w \rightarrow a}\left(s_{D}(z, w)-s_{\Pi}(\eta(z), \eta(w))=0 .\right.
$$

Hence

$$
\liminf _{z, w \rightarrow a}\left(c_{D}(z, w)-s_{D}(z, w)\right) \geq 0 .
$$

It remains to show that

$$
\limsup _{z, w \rightarrow a}\left(k_{D}(z, w)-s_{D}(z, w)\right) \leq 0 .
$$

For this, we choose a Dini-smooth simply connected domain $F \subset D$ such that $\partial F=\partial D$ near $a$. Then we may proceed similarly to above.

In the next two proofs we shall use the quasi-hyperbolic distance $h_{D}$, i.e. the integrated form of $1 / d_{D}$.

Proof of Proposition [6 for $b_{D}$. Let $\varepsilon \in(0,1 / 4]$. The arguments from the previous proof and Lemma $\mathrm{L}$ implies that there exist neighborhoods $U_{2} \subset U_{1}$ of $a$ such that $F=D \cap U_{1}$ is a Dini-smooth simply connected domain and $\beta_{D}>\beta_{F}-\varepsilon$ on $D \cap U_{2}$. 
Let $z, w \in D$. We may choose a smooth curve $\gamma:[0,1] \rightarrow D$ with $\gamma(0)=z, \gamma(1)=w$ and

$$
b_{D}(z, w)+\varepsilon>\int_{0}^{1}\left(\beta_{D}(\gamma(t))|d \gamma(t)| .\right.
$$

It follows by the proof of [10, Proposition 1] that one may find a neighborhood $U_{3} \subset U_{2}$ of $a$ such that $\gamma \subset D \cap U_{2}$ if $z, w \in D \cap U_{3}$.

Let $\psi: F \rightarrow \mathbb{D}$ be a Riemann map, $\tilde{\gamma}=\psi \circ \gamma, z, w \in D \cap U_{3}$ and $\tilde{z}=\psi(z), \tilde{w}=\psi(w)$. Using that $\varepsilon \leq 1 / 4$, we obtain that

$$
\begin{aligned}
\sqrt{2}\left(b_{D}(z, w)+\varepsilon\right) & >\sqrt{2} \int_{0}^{1}\left(\beta_{\mathbb{D}}(\psi(t))-\varepsilon\right)|d \psi(t)|>\int_{0}^{1} \frac{|d \psi(t)|}{d_{\mathbb{D}}(\psi(t))} \\
& \geq h_{\mathbb{D}}(\tilde{z}, \tilde{w}) \geq 2 k_{\mathbb{D}}(\tilde{z}, \tilde{w})-c|\tilde{z}-\tilde{w}|=: 2 \tilde{s}_{D}(z, w),
\end{aligned}
$$

where $c>0$ is a constant. Since $\lim _{z, w \rightarrow a}\left(s_{D}(z, w)-\tilde{s}_{D}(z, w)\right)=0$, we get

$$
\liminf _{z, w \rightarrow a}\left(b_{D}(z, w)-\sqrt{2} s_{D}(z, w)\right) \geq 0 .
$$

The proof of the opposite inequality

$$
\limsup _{z, w \rightarrow a}\left(b_{D}(z, w)-\sqrt{2} s_{D}(z, w)\right) \leq 0 .
$$

is even simpler. We choose $U_{2}$ such that $\beta_{D}<\beta_{F}+\varepsilon$ on $D \cap U_{2}$. Then we may take $U_{3}$ such that the $b_{F}$-geodesic for any $z, w \in D \cap U_{3}$ belongs to $D \cap U_{2}$. It follows that $b_{D}(z, w) \leq b_{F}(z, w)+c^{\prime}|z-w|$ for some constant $c^{\prime}>0$ which implies (1).

Proof of Proposition 7 . (a) First, we shall show that

$$
\lim _{\substack{z, w \rightarrow a \\ z \neq w}} \frac{c_{D}(z, w)}{k_{D}(z, w)}=1 .
$$

We may proceed as in the proof of Proposition 6 , choosing $\zeta$ to be a $C^{1}$-smooth curve and then observing that $\psi$ extends to a homeomorphism from $\bar{G}$ to $\overline{\mathbb{D}}$. Then there exists a disc $U$ centered at $\eta(a)$ such that $\eta(D) \subset \mathbb{D} \cap U$. Hence

$$
1 \geq \frac{c_{D}(z, w)}{k_{D}(z, w)} \geq \frac{c_{\mathbb{D}}(\eta(z), \eta(w))}{k_{\mathbb{D} \cap U}(\eta(z), \eta(w))}, \quad z \neq w .
$$

It follows similarly to the proof of Lemma $L$ that the last quotient tends to 1 as $z, w \rightarrow a$.

Second, we have that (see [4])

$$
\lim _{z \rightarrow a} 2 \kappa_{D}(z) d_{D}(z)=\sqrt{2} \beta_{D}(z) d_{D}(z)=1 .
$$


It follows as in the proof of [9, Proposition 5] that

$$
\lim _{\substack{z, w \rightarrow a \\ z \neq w}} \frac{h_{D}(z, w)}{p_{D}(z, w)}=2 .
$$

It remains to use that, by [9, Proposition 6 (a)],

$$
\lim _{\substack{z, w \rightarrow a \\ z \neq w}} \frac{h_{D}(z, w)}{s_{D}(z, w)}=2 .
$$

(b) It is a direct consequence of (a) for $k_{D}$ and $b_{D}$, since they are inner distances. On the other hand, by [7, Proposition 9],

$$
\lim _{z \rightarrow \partial D} \frac{c_{D}(z, w)}{k_{D}(z, w)}=1 \quad \text { uniformly in } w \neq z
$$

which completes the proof.

\section{REFERENCES}

[1] Z.M. Balogh, M. Bonk, Gromov hyperbolicity and the Kobayashi metric on strictly pseudoconvex domains, Comment. Math. Helv. 75 (2000), 504-533.

[2] K. Diedercih, J.E. Fornaess, Boundary behavior of the Bergman metric, arXiv:1504.02950.

[3] S. Fu, Asymptotic expansions of invariant metrics of strictly pseudoconvex domains, Canad. Math. Bull. 38 (1995), 196-206.

[4] M. Jarnicki, N. Nikolov, Behavior of the Carathéodory metric near strictly convex boundary points, Univ. Iag. Acta Math. XL (2002), 7-12.

[5] M. Jarnicki, P. Pflug, Invariant distances and metrics in complex analysis - 2nd extended edition, de Gruyter, Berlin, 2013.

[6] D. Ma, Sharp estimates for the Kobayashi metric near strongly pseudoconvex points, Contemp. Math. 137 (1992), 329-338.

[7] N. Nikolov, Estimates of invariant distances on "convex" domains, Ann. Mat. Pura Appl. 193 (2014), 1595-1605.

[8] N. Nikolov, Comparison of invariant functions on strongly pseudoconvex domains, J. Math. Anal. Appl. 421 (2015), 180-185.

[9] N. Nikolov, L. Andreev, Estimates of the Kobayashi and quasi-hyperbolic distances, arXiv:1510.01571.

[10] N. Nikolov, M. Trybuła, Estimates of the Bergman distance on Dini-smooth bounded planar domains, Collect. Math., DOI: 10.1007/s13348-015-0150-2.

[11] Ch. Pommerenke, Boundary behaviour of conformal maps, Springer, Berlin, 1992.

N. Nikolov: Institute of Mathematics and Informatics, Bulgarian Academy of Sciences, Acad. G. Bonchev 8, 1113 Sofia, Bulgaria

Faculty of Information Sciences, State University of Library StudIES And Information Technologies, Shipchenski Prohod 69A, 1574 SofiA, BULGARIA

E-mail address: nik@math.bas.bg 
BOUNDARY BEHAVIOR OF INVARIANT FUNCTIONS ON PLANAR DOMAINS 9

M. Trybula: Faculty of Mathematics and Informatics, Adam Mickiewicz University, Umultowska 87, 61-001 Poznań, Poland

E-mail address: maria.h.trybula@gmail.com

L. Andreev: Institute of Mathematics and Informatics, Bulgarian Academy of Sciences, Acad. G. Bonchev 8, 1113 Sofia, Bulgaria,

E-mail address: lyubomir.andreev@math.bas.bg 